University of South Carolina

Scholar Commons

2-19-2001

\title{
Diamagnetic Persistent Current in Diffusive Normal-Metal Rings
}

E. M.Q. Jariwala

P. Mohanty

M. B. Ketchen

Richard A. Webb

University of South Carolina - Columbia, webbra@mailbox.sc.edu

Follow this and additional works at: https://scholarcommons.sc.edu/phys_facpub

Part of the Physics Commons

\section{Publication Info}

Published in Physical Review Letters, Volume 86, Issue 8, 2001, pages 1594-1597.

Jariwala, E.M.Q., Mohanty, P., Ketchen, M.B., and Webb, R.A. (2001). Diamagnetic Persistent Current in Diffusive Normal-Metal Rings. Physical Review Letters, 86(8), 1594-1597. doi: 10.1103/

PhysRevLett.86.1594

(C) 2001 The American Physical Society.

This Article is brought to you by the Physics and Astronomy, Department of at Scholar Commons. It has been accepted for inclusion in Faculty Publications by an authorized administrator of Scholar Commons. For more information, please contact digres@mailbox.sc.edu. 


\title{
Diamagnetic Persistent Current in Diffusive Normal-Metal Rings
}

\author{
E. M. Q. Jariwala, ${ }^{1}$ P. Mohanty, ${ }^{1} *$ M. B. Ketchen, ${ }^{2}$ and R. A. Webb ${ }^{1}$ \\ ${ }^{1}$ Center for Superconductivity Research, Department of Physics, \\ University of Maryland, College Park, Maryland 20742 \\ ${ }^{2}$ IBM T.J. Watson Research Center, Yorktown Heights, New York 10598
}

(Received 9 December 1999)

\begin{abstract}
We have measured a diamagnetic persistent current with flux periodicities of both $h / e$ and $h / 2 e$ in an array of thirty diffusive mesoscopic gold rings. At the lowest temperatures, the magnitudes of the currents per ring corresponding to the $h / e$ - and $h / 2 e$-periodic responses are both comparable to the Thouless energy $E_{c} \equiv \hbar / \tau_{D}$, where $\tau_{D}$ is the diffusion time. Taken in conjunction with earlier experiments, our results strongly challenge the conventional theories of persistent current. We consider a new approach associated with the saturation of the phase coherence time $\tau_{\varphi}$.
\end{abstract}

DOI: $10.1103 /$ PhysRevLett.86.1594

The phase of the electron wave function lies at the heart of many mesoscopic phenomena. Consider a phasecoherent ring of length $L$ : the presence of an AharonovBohm flux $\Phi$ introduces a phase factor into the boundary condition $\Psi(x+L)=\Psi(x) e^{2 \pi i \Phi / \Phi_{0}}$, such that all thermodynamic quantities are oscillatory in the applied flux, with a period $\Phi_{0}=h / e$. To minimize its free energy $F$, this isolated ring will support an equilibrium persistent current $I=-\partial F / \partial \Phi$, even in the presence of disorder [1]. Moreover, for an ensemble of such rings, the fundamental harmonic of this current (with flux periodicity $h / e$ ) is strongly suppressed, whereas the next harmonic (with flux periodicity $h / 2 e$ ) survives due to the contribution of time-reversed paths of the electron [2].

Nanoampere-sized $h / e$ - or $h / 2 e$-periodic currents have been separately observed in a few experiments: the $h / e$ current has been measured in single Au rings [3] and in a GaAs-AlGaAs ring [4], while the $h / 2 e$ current has been measured in multiring arrays of $10^{7} \mathrm{Cu}$ rings [5] and $10^{5}$ GaAs-AlGaAs rings [6], where the $h / e$ response was not observable. Except for the case of the nearly ballistic GaAs ring [4], all the currents measured are remarkably 10-100 times larger than theoretically expected [7-9]. The diamagnetic sign of the average $h / 2 e$ response at high frequencies in Ref. [6] also contrasts with most predictions. Disorder plays a strong role in these results, emphasizing the Thouless scale of electron diffusion $E_{c}$.

In this Letter, we report the first experiment on a single sample to measure the sign, amplitude, and temperature dependence for both the $h / e$ and $h / 2 e$ components of the persistent current. For our array of 30 diffusive $\mathrm{Au}$ rings, we determine the sign of both currents to be diamagnetic near zero field. At our lowest temperature of $5.5 \mathrm{mK}$, the amplitudes of the total $h / e$ and $h / 2 e$ currents are $\left\langle I_{1 T}\right\rangle=1.92 \mathrm{nA}$ and $\left\langle I_{2 T}\right\rangle=1.98 \mathrm{nA}$, respectively. For comparison to previous experiments, we present our results for the currents per ring as $\left\langle I_{1}\right\rangle=\left\langle I_{1 T}\right\rangle / \sqrt{30}=$ $0.35 \mathrm{nA}=2.3 e E_{c} / \hbar$ and $\left\langle I_{2}\right\rangle=\left\langle I_{2 T}\right\rangle / 30=0.066 \mathrm{nA}=$ $0.44 e E_{c} / \hbar$. We fit the temperature dependence of both the $h / e$ and $h / 2 e$ currents to the form $e^{-T / T_{0}}$ [8], as well as
PACS numbers: 73.23.Ra, 05.40.-a, 73.50.Mx

to $e^{-\sqrt{T / T_{0}}}$, and compare the different characteristic temperatures $T_{0}$ to $E_{c}$. The sum of all the experimental results prompts a discussion of new calculations based on a direct connection to the saturation of $\tau_{\varphi}$ at low temperatures.

Figure 1(a) displays a section of our array of $30 \mathrm{Au}$ rings, fabricated using $e$-beam lithography on top of one arm of the counterwound $\mathrm{Nb}$ pickup coil of a dc SQUID. A 150-nm-thick layer of $\mathrm{SiO}$ separates the coils from the $\mathrm{Au}$ rings. We compute that the mutual inductance of each ring to the coil is $2.7 p \mathrm{H}$ and uniform to within $5 \%$. The gradiometer configuration of the two counterwound coils is clearly displayed in Fig. 1(b) together with the pair of on-chip $\mathrm{Nb}$ field coils. Operating the dc SQUID in a fluxlocked loop mode, we measure a typical flux noise of

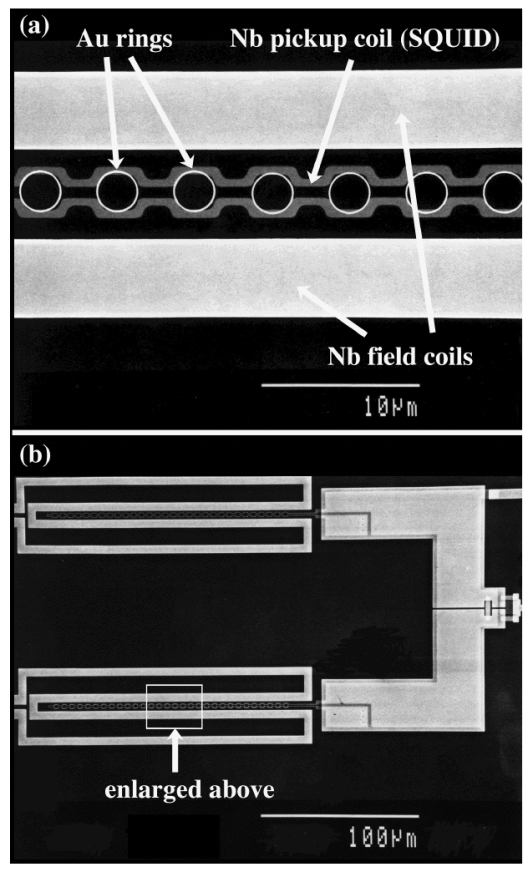

FIG. 1. (a) Electron micrograph showing the Au rings, as well as the pickup and field coils of the SQUID. (b) Larger view illustrating the entire gradiometer design of the SQUID. 
$\sim 3 \mu \Phi_{S} / \sqrt{\mathrm{Hz}}$ down to $2 \mathrm{~Hz}$ at low temperatures. Here $\Phi_{S}$ denotes the superconducting flux quantum.

Each ring has a perimeter of length $L=8.0 \pm 0.2 \mu \mathrm{m}$, with a line thickness $t=60 \pm 2 \mathrm{~nm}$ and linewidth $w=120 \pm 20 \mathrm{~nm}$. We estimate $R_{\square}=0.15 \Omega / \square$ and a diffusion constant of $D=v_{F} l_{e} / 2=0.06 \mathrm{~m}^{2} / \mathrm{s}$, where the elastic mean free path $l_{e} \simeq 87 \mathrm{~nm}$. These parameters as well as the phase coherence length $L_{\varphi} \equiv \sqrt{D \tau_{\varphi}}$ are measured separately in a $207 \mu \mathrm{m}$ long meander with the same line thickness and line width as the rings. By using a standard weak localization measurement of the magnetoresistance, we determined that $L_{\varphi} \approx 16 \mu \mathrm{m}$ for this control sample and is temperature independent below $500 \mathrm{mK}$ [10]. The Thouless energy $E_{c} \equiv \hbar / \tau_{D}=\hbar D / L^{2}$ is $7.3 \mathrm{mK}$; the mean energy level spacing is given by $\Delta \equiv 1 / 2 \mathcal{N}_{0} V=19 \mu \mathrm{K}$, where $\mathcal{N}_{0}$ is the density of states and $\mathcal{V}$ is the volume of each ring.

We measure the response of the rings to an applied magnetic field as a change in the average magnetic moment coupled to the SQUID pickup coil. To distinguish the flux-periodic current in the rings from the background magnetization, we modulate the field at a small frequency $f$ and measure separately the first, second, and third harmonics at $f, 2 f$, and $3 f$, respectively. The amplitudes of the periodic signals measured are related to the ac modulation amplitude by a Bessel function, allowing us to maximize the signal in a particular harmonic for either the $h / e$ or the $h / 2 e$ flux-periodic response [11]. The response is independent of the measurement frequency $f$ below $12 \mathrm{~Hz}$, as also seen previously [3].

In the following, we discuss a typical data set, shown in Fig. 2, which focuses on the $h / 2 e$ oscillations in the second harmonic of the magnetization using a $2.26 \mathrm{G}$ ac modulation. As in most of our runs, we apply this ac field at $f=2.02 \mathrm{~Hz}$, which is superimposed on a dc field. The dc field is swept slowly over $\pm 20 \mathrm{G}$ at $3.6 \mathrm{G} / \mathrm{h}$. The data is averaged in $0.08 \mathrm{G}$ bins for over $80 \mathrm{~s}$ per bin, resulting in a noise floor of $\sim 0.3 \mu \Phi_{\mathrm{S}}$ for each bin. In Fig. 2(a) we display the raw data (dashes) of the second harmonic component at frequency $4.04 \mathrm{~Hz}$ taken at the base temperature of $5.5 \mathrm{mK}$, along with the $h / 2 e$-periodic contribution (solid line) represented by the signal in the $h / 2 e$ window of the power spectrum shown in Fig. 2(b). The width of these windows corresponding to $h / e$ - or $h / 2 e$ periodic currents reflect the finite linewidth of the rings; furthermore, they are broadened to account for the effect of the intrinsic width of the transform due to the finite field range of the data. The autocorrelation function of the raw data is displayed in Fig. 2(c), clearly identifying an oscillatory function in flux corresponding to an $h / 2 e$-periodic response from the rings. Similar measurements maximizing the $h / 2 e$ response in the third harmonic at $6.06 \mathrm{~Hz}$ yield similar results. One previously unreported observation is that the amplitude of the periodic response appears to decrease exponentially as $e^{-H / H_{c}}$ with $H_{c} \approx 6 \mathrm{G}$. If this decay is due to the dephasing effect of magnetic field penetration into the arms of the ring [12], then our observa-
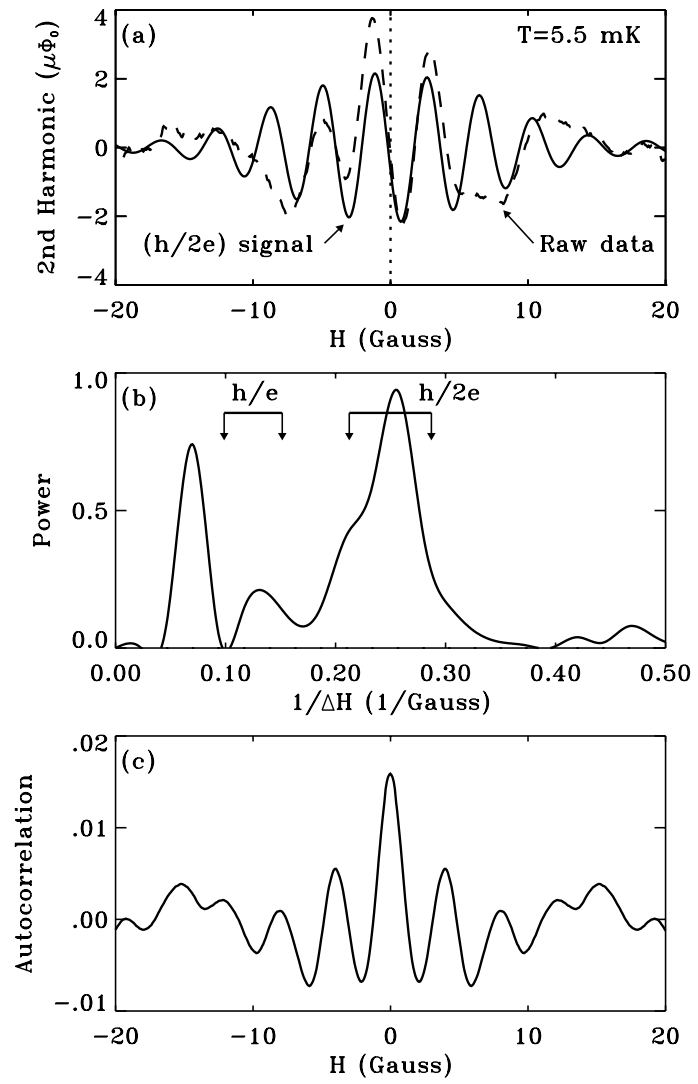

FIG. 2. Magnetic response of $30 \mathrm{Au}$ rings from a run focusing on $h / 2 e$ oscillations. The dashed line in (a) represents raw data. The solid line represents the $h / 2 e$-periodic contribution, taken from the $h / 2 e$ window in the power spectrum in (b). The autocorrelation shown in (c) also demonstrates the $h / 2 e$ periodic signal.

tion is consistent with the corresponding weak-localization field scale of $H_{c}=\sqrt{3} \hbar / e w L$.

A similar decaying envelope is seen in the $h / e$-periodic response, as shown in Fig. 3(a). Here we use a $5.38 \mathrm{G} \mathrm{ac}$ modulation to maximize the $h / e$ oscillations in the third harmonic at $6.06 \mathrm{~Hz}$. The solid line in Fig. 3(a) represents the $h / e$-periodic contribution to the total signal. Note that the leftmost peak shown in the power spectrum in both Figs. 2(b) and 3(b) results from the finite field range of our data with a background subtracted. The size of our rings has been chosen to avoid confusion of the $h / e$ - or $h / 2 e$-periodic signals with this lower-frequency response.

The data in Figs. 2(a) and 3(a) also illustrate the diamagnetic sign of the response near zero field. This determination depends inherently on the exact wiring of the SQUID chip and electronics, but is confirmed empirically by comparing an applied phase-modulated magnetic field with the sign of the SQUID response in the higher harmonics. Both the $h / 2 e$ and the $h / e$ responses are consistently diamagnetic for all ac field drives used, at all temperatures investigated, and on at least three different coldowns.

Figure 4 displays the temperature dependence of the total $h / 2 e$ and $h / e$ currents up to $150 \mathrm{mK}$. The values for current are calculated from the flux response using the 

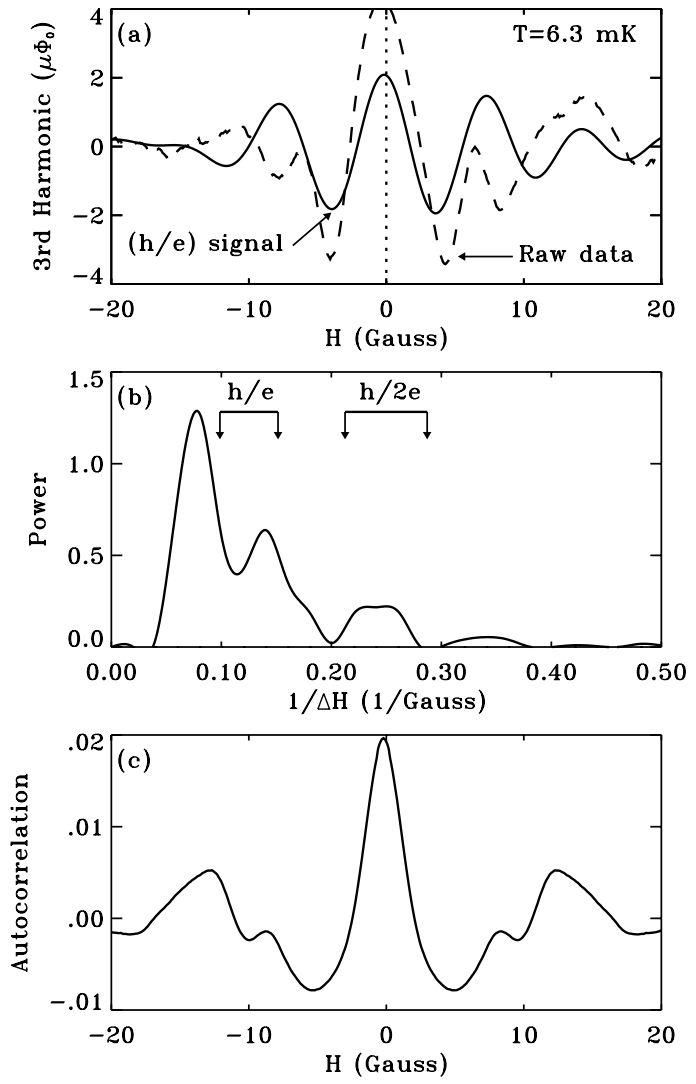

FIG. 3. Magnetic response of $30 \mathrm{Au}$ rings from a run focusing on $h / e$ oscillations. The dashed line in (a) represents raw data. The solid line represents the $h / e$-periodic contribution, taken from the $h / e$ window in the power spectrum in (b). The autocorrelation is shown in (c).

mutual inductance and the appropriate Bessel function factor [11]. The solid lines drawn through the data are fits to $I=I_{0} e^{-T / T_{0}}$ and we find $T_{0}=89$ and $166 \mathrm{mK}$ for the $h / 2 e$ and $h / e$ responses, respectively. Our data can also be fit over this limited temperature range to $I \propto e^{-\sqrt{T / T_{0}}}$ with $T_{0}=25 \mathrm{mK}$ for the $h / 2 e$ signal and $50 \mathrm{mK}$ for the $h / e$ signal. Theoretically it is expected that $T_{0} \propto E_{c}$ for both functional forms $[7,8]$, but the comparison of our $T_{0}$ with previous experiments suggests that this may not be the correct energy scale. For example, our $T_{0}$ for the $h / 2 e$ signal is quite similar to that obtained in the $\mathrm{Cu}$ array experiment [5], though their $E_{c}$ was 3.3 times larger than ours. A similar discrepancy arises in comparing the temperature dependence of the $h / e$ signal in a single ring [3] to our present result. At least for our data, we can say that the $h / 2 e$ response is more strongly temperature dependent, with $T_{0}$ about half that for the $h / e$ response given either exponential functional form.

The sign of the persistent current, along with its magnitude at $T=0$, is an integral part of most theories for the $h / 2 e$ or the $h / e$ response. At our lowest $T=5.5 \mathrm{mK}$, we measure a diamagnetic $h / 2 e$ current of $\left\langle I_{2}\right\rangle=\left\langle I_{2 T}\right\rangle / 30=$ $0.066 \mathrm{nA}$, dividing the total response by the number of rings to obtain the average current per ring. Corresponding to $\left\langle I_{2}\right\rangle=0.44 e E_{c} / \hbar$, our result is similar to the value
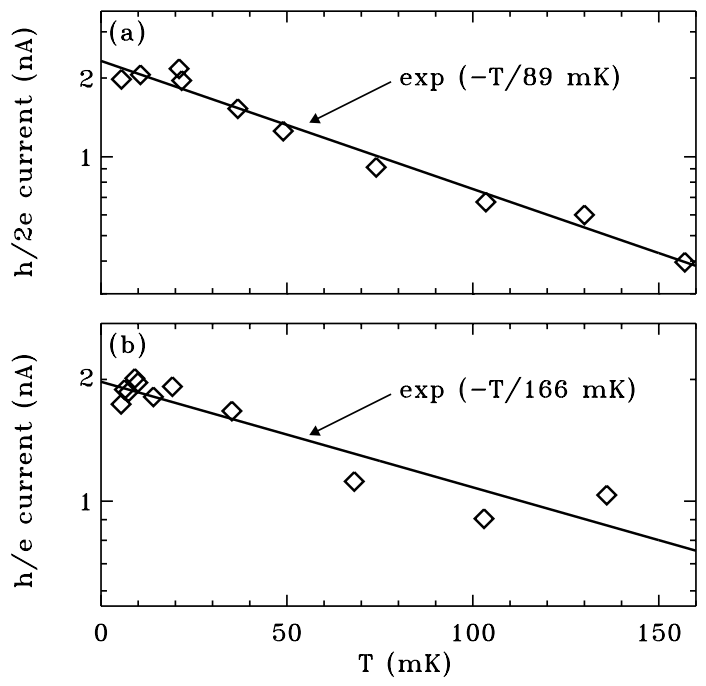

FIG. 4. Temperature dependences of the total (a) $h / 2 e$ and (b) $h / e$ currents calculated from the net magnetic response of the $30 \mathrm{Au}$ rings. The solid lines are fits to the form $\exp \left(-T / T_{0}\right)$.

of $0.77 e E_{c} / \hbar$ measured in a much larger ensemble of diffusive rings [5]. Both of these results are comparable to the sample-specific typical current $I^{\text {typ }}$ predicted for a single ring (which should vanish in an array of rings), but are nearly 2 orders of magnitude larger than the expected value of $\sim e \Delta / \hbar$ for the ensemble-averaged $h / 2 e$ current [7,9]. Electron-electron interactions yield an $h / 2 e$-periodic average current within an order of magnitude of $e E_{c} / \hbar$, but the sign is required to be paramagnetic [8]. Substituting an attractive phonon-mediated interaction finally yields the correct diamagnetic sign but reduces the amplitude in Ref. [8] by 1 order of magnitude [13]. For the $h / 2 e$ persistent current, the combined result of the $\sim e E_{c} / \hbar$ magnitude (also in Refs. [5,6]) and the diamagnetic sign (also in Ref. [6]) disagrees with all conventional theories.

For the $h / e$ response, the diamagnetic sign and the magnitude also present a challenge. The typical $h / e$ current in any one ring is sample specific but of order $I^{\text {typ }} \sim e E_{c} / \hbar$ [7]; a previous experiment detected paramagnetic $h / e$ currents almost 100 times larger, though, in two different rings [3]. For our experiment on an array of $N=30$ rings, we measure an $h / e$ current per ring of $\left\langle I_{1}\right\rangle=\left\langle I_{1 T}\right\rangle / \sqrt{30}=0.35 \mathrm{nA}$ at $5.5 \mathrm{mK}$, where we have divided by $\sqrt{N}$ to account for the random sign of the typical current. Corresponding to $\left\langle I_{1}\right\rangle=2.3 e E_{c} / \hbar$, our result is much closer to the value $I^{\text {typ }}$ predicted for a single ring. Alternatively, if the diamagnetic $h / e$ signal from our array comes from the contribution of currents all with the same sign, then dividing our total $h / e$ response by $N$ instead of $\sqrt{N}$ yields a value $\left\langle I_{1}\right\rangle \lesssim I^{\text {typ }}$ for the mean current per ring, but over 100 times larger than the more appropriate ensemble-averaged calculation of $e \Delta / \hbar$ [9]. Regardless of this question over the exact distribution of signs and amplitudes, the presence of a large $h / e$ signal in our array suggests that $N=30$ rings are not enough for ensemble averaging, as in Refs. [5,6]. New experiments 
on different arrays are needed here to clarify the distinction between the ensemble average and the typical currents in a finite array of rings.

The significant gap between the $h / 2 e$ persistent current experiments and theory [14] suggests we look elsewhere for a solution. It is known that ac noise in a mesoscopic conductor can produce both a rectified dc current [15] and decoherence of the electron wave function [12]. Reference [16] predicts that in a closed loop this noise induced diamagnetic dc current is on the order of $e / \tau_{D}=e D / L^{2}$, within a factor of 2 of the experimental $h / 2 e$ persistent current values. The high-frequency noise here can be from external incoherent sources or can arise internally, e.g. from two level systems [17], 2-channel Kondo impurities [18], or nuclear spins. It was recently suggested $[19,20]$ that this same ac noise may also explain the theoretically unexpected saturation of the dephasing time $\tau_{\varphi}$ at low temperatures [10]. In fact, Ref. [20] gives a universal relationship $\left\langle I_{2}\right\rangle=\mathrm{Ce} / \tau_{0}$ (where the constant $C$ of order 1 also contains the sign), connecting the ensemble-averaged $h / 2 e$ current $\left\langle I_{2}\right\rangle$ to the dephasing time $\tau_{0}$ at $T=0$ via the same nonequilibrium noise. Estimating $\tau_{0} \approx 4.2 \mathrm{~ns}[10]$ and accounting for strong spin-orbit scattering in our gold rings, this formula yields a value of $0.025 \mathrm{nA}$ but paramagnetic in sign, as compared to our diamagnetic measurement of $0.066 \mathrm{nA}$ per ring. For larger arrays of rings with weaker spin-orbit scattering, this new idea appears to also give the correct $h / 2 e$ amplitude based on $\tau_{0}[5,6]$, as well as the observed diamagnetic sign [6]. We add that energy averaging effects may explain why the persistent current continues to grow below the temperature at which $\tau_{\varphi}$ saturates. We also believe that the origin of this ac noise must be internal to the sample because recent dephasing measurements in the presence of external ac noise [21] cannot explain the magnitude or saturation behavior of $\tau_{\varphi}$ in Au wires.

In summary, we have presented the first temperature dependent measurements of both the $h / 2 e$ and $h / e$ components of the persistent current from an array of 30 diffusive Au rings. The magnitude of our $h / 2 e$ response corresponds to an average current per ring of $\sim e E_{c} / \hbar$, in agreement with previous experiments in multiple-ring systems. The magnitude of our $h / e$ current is also found to be $\sim e E_{c} / \hbar$. We find a diamagnetic response for both the $h / e$ and $h / 2 e$ signals. These results, including the origin of $T_{0}$ in the temperature dependence, cannot be explained within the framework of existing theories. The new suggestion that the $h / 2 e$ current and the saturation of $\tau_{\varphi}$ result from the same internal ac noise appears promising but needs to be explored more carefully.

We thank B. L. Altshuler, V. Ambegaokar, H. Bouchiat, Y. Gefen, Y. Imry, V.E. Kravtsov, D. Mailly, G. Montambaux, P. Schwab, J. Schwarz, R. Smith, and T.R. Stevenson for discussions. This work is supported by the NSF under Contract No. DMR 97-30577 and by the ARO under Contract No. DAAG 55-97-10330.

*Present address: Dept. of Phys., Cond. Mat. Div. 114-36, California Institute of Technology, Pasadena, California 91125.

[1] N. Byers and C. N. Yang, Phys. Rev. Lett. 7, 46 (1961); F. Bloch, Phys. Rev. B 2, 109 (1970); M. Buttiker, Y. Imry, and R. Landauer, Phys. Lett. 96A, 365 (1983).

[2] H. Bouchiat and G. Montambaux, J. Phys. (Paris) 50, 2695 (1989); G. Montambaux et al., Phys. Rev. B 42, 7647 (1990).

[3] V. Chandrasekhar et al., Phys. Rev. Lett. 67, 3578 (1991).

[4] D. Mailly, C. Chapelier, and A. Benoit, Phys. Rev. Lett. 70, 2020 (1993).

[5] L.P. Levy et al., Phys. Rev. Lett. 64, 2074 (1990); L. P. Levy, Physica (Amsterdam) 169B, 245 (1991).

[6] B. Reulet et al., Phys. Rev. Lett. 75, 124 (1995).

[7] H. F. Cheung, E. K. Riedel, and Y. Gefen, Phys. Rev. Lett. 62, 587 (1989).

[8] V. Ambegaokar and U. Eckern, Phys. Rev. Lett. 65, 381 (1990); 67, 3192 (1991); U. Eckern, Z. Phys. B 82, 393 (1991).

[9] A. Schmid, Phys. Rev. Lett. 66, 80 (1991); F. von Oppen and E. K. Riedel, ibid. 66, 84 (1991); B.L. Altshuler, Y. Gefen, and Y. Imry, ibid. 66, 88 (1991).

[10] P. Mohanty, E. M. Q. Jariwala, and R. A. Webb, Phys. Rev. Lett. 78, 3366 (1997). Our control sample is "Au-2."

[11] The exact Bessel function relation is described in Ref. [5].

[12] B. L. Altshuler and A. G. Aronov, in Electron-Electron Interactions in Disordered Systems, edited by A. L. Efros and M. Pollak (Elsevier, Amsterdam, 1985); S. Chakravarty and A. Schmid, Phys. Rep. 140, 193 (1986).

[13] V. Ambegaokar and U. Eckern, Europhys. Lett. 13, 733 (1990).

[14] U. Eckern and P. Schwab, Adv. Phys. 44, 387 (1995).

[15] V. I. Fal'ko and D.E. Khmelnitskii, Zh. Eksp. Teor. Fiz. 95, 328 (1989) [Sov. Phys. JETP 68, 186 (1989)].

[16] V.E. Kravtsov and V. I. Yudson, Phys. Rev. Lett. 70, 210 (1993).

[17] Y. Imry, H. Fukuyama, and P. Schwab, Europhys. Lett. 47, 608 (1999).

[18] A. Zawadowski, J. von Delft, and D. C. Ralph, Phys. Rev. Lett. 83, 2632 (1999).

[19] P. Mohanty, in special issue on The Proceedings of Localization-1999, Hamburg, Germany, edited by M. Schreiber [Ann. Phys. (Leipzig) 8, 549 (1999)].

[20] V.E. Kravtsov and B.L. Altshuler, Phys. Rev. Lett. 84, 3394 (2000).

[21] R.A. Webb et al., in Quantum Coherence and Decoherence-Proceedings of ISQM Tokyo 1998, edited by K. Fujikawa and Y. A. Ono (Elsevier, Amsterdam, 1999). 